\title{
Hyperglycemia in the intensive care unit: is insulin the only option?
}

Kathleen M Dungan

See related research by Christiansen et al., http://ccforum.com/content/17/5/R192

\begin{abstract}
Current guidelines advocate the use of insulin for the management of hyperglycemia in the hospital setting. However, insulin is limited by a narrow therapeutic window, frequent errors, a need for expertise and systems-based monitoring, and lack of specificity for metabolic abnormalities that occur during critical illness. As a result, non-insulin alternatives have garnered increasing interest for managing hyperglycemia in the hospital. However, non-insulin therapies have had safety and tolerability concerns, patients may still need insulin for glycemic control, and there have been limited outcomes data supporting their use. In the study by Christiansen and colleagues in the previous issue of Critical Care, pre-admission metformin therapy was associated with reduced mortality in critically ill patients with type 2 diabetes. The mortality benefit persisted after controlling for other variables, and was particularly prominent when metformin was continued during admission. Furthermore, the reduction in mortality was observed despite a slightly increased prevalence of lactic acidosis in metformin users. The protective effects of metformin are purported to be related to pleiotropic, possibly anti-inflammatory mechanisms, raising the question of benefit in patients without diabetes. Thus, the findings warrant a re-appraisal of the risks and benefits of metformin use during critical illness. However, in order to justify the revision of multiple guidelines and changes in product labeling, clinical trials in carefully selected patient populations are indicated.
\end{abstract}

Correspondence: kathleen.dungan@osumc.edu

5th Floor McCampbell Hall, Division of Endocrinology, Diabetes and Metabolism, Ohio State University, 1581 Dodd Drive, Columbus, OH 43210, USA

\section{Introduction}

Appropriate glucose targets and management strategies among hospitalized patients with hyperglycemia have been the focus of much debate. In the previous issue of Critical Care, Christiansen and colleagues [1] present data suggesting the need for a re-appraisal of metformin therapy in the ICU. Using multivariable analyses, the retrospective cohort study demonstrates reduced mortality in critically ill patients with type 2 diabetes receiving pre-admission metformin therapy. Confounding by indication is minimized through multiple sensitivity analyses, and even more intriguing is the observation that continued use throughout ICU stay was associated with greater mortality benefit than interrupting metformin at admission (hazard ratios of 0.25 and 0.67 , respectively). Although the mechanism is unclear, it is hypothesized that pleiotropic, possibly non-glycemic, anti-inflammatory effects of metformin may be responsible for its mortality benefit. The tantalizing conclusion to be drawn from this study would be to recommend continuation of metformin therapy in all hospitalized patients with type 2 diabetes receiving pre-admission metformin therapy and possibly to explore its use in other select ICU patients. However, these conclusions are still premature and have not yet been confirmed, despite a similar report in postcardiac surgery patients [2].

\section{Treatment approaches for hyperglycemia in the intensive care unit}

Lactic acidosis is the primary motivator for recommendations to discontinue metformin at hospital admission [3,4]. Christiansen and colleagues report that lactic acidosis was limited to metformin users only but was still uncommon (10 patients, $0.4 \%$ ) given the multiple risk factors likely to be present during critical illness. By comparison, the rate of lactic acidosis reported in the product labeling is 0.03 cases per 1,000 patient-years [5], although with approved prescribing patterns, it is virtually undetectable [6]. Nevertheless, mortality from metformin-associated lactic acidosis is reportedly as high as $50 \%$ [5]. Given the potential benefit 
reported here (and elsewhere), it seems that the risk of lactic acidosis might be addressed through a case-finding approach rather than by eliminating its use altogether in the hospital [6]. Unfortunately, clinicians are still constrained by prescribing restrictions [7], particularly in the US [5], despite calls for more relaxed or individualized dosing or both $[7,8]$.

In contrast, guidelines for hospital management of hyperglycemia have recommended insulin as the preferred treatment modality for a variety of reasons $[3,4]$. It is highly effective, quickly titrated to goal, and has very few contraindications. However, both intravenous and subcutaneous insulin require complicated dosing regimens which invite clinical inertia and overreliance on 'sliding scale' insulin in the absence of sufficient resources or expertise. Errors are common and lead to harm more frequently than errors from other drugs $[9,10]$. Moreover, the indiscriminate interruption of stable home regimens in favor of insulin at the time of hospital admission has the potential for creating confusion at discharge, including omission of glucose-lowering therapy altogether [11]. Insulin also has a narrow therapeutic window, increasing the risk of hypoglycemia. Although the precise causal relationship between hypoglycemia and mortality is debated, hypoglycemia certainly causes harm and has impeded efforts to determine whether tight glycemic control is of any value in the ICU [12]. Finally, insulin does not directly address the pathophysiologic abnormalities that are characteristic of stress hyperglycemia [13].

On the other hand, metformin carries minimal risk of hypoglycemia, is simple to administer, and the mechanism of action is more strategically aligned with pathophysiologic abnormalities observed during critical illness. In addition to metformin, other agents, particularly glucagon-like peptide-1 receptor agonists and dipeptidyl peptidase-IV inhibitors, have received attention as possible alternatives or adjuncts to insulin therapy in the hospital [14]. However, these agents have also raised safety or tolerability concerns [15], and it is unknown whether these agents affect outcomes during critical illness. Importantly, neither metformin nor other non-insulin therapies are likely to preclude the need for insulin to maintain glycemic control in some patients.

Nevertheless, a rational approach to the study of metformin might justify its use in select cases in the hospital, where it can be shown that the benefits outweigh the risks of lactic acidosis. Given the deeply entrenched sentiments (deserved or not) about the risk of lactic acidosis, it hardly seems prudent to recommend the routine initiation or even continuation of metformin in most critically ill patients without data from a carefully designed clinical trial. Owing to the observed heterogeneity of effects among various disease states and the hypothesized anti-inflammatory mechanism of action, a clinical trial would preferably be performed among more targeted disease populations such as patients with sepsis. Further study should also address the role of chronic hyperglycemia, which not only appears to engender less harm than acute hyperglycemia in the ICU setting [13] but also appears to abrogate some of the mortality benefit of metformin in this study [1].

\section{Conclusions}

In conclusion, recent data further solidify the use of metformin as first-line treatment for type 2 diabetes. Given the limitations of insulin therapy and mounting evidence for a protective effect of metformin, carefully conducted clinical trials in the hospital setting are indicated.

\section{Competing interests}

The author reports research support from Novo Nordisk (Bagsvaerd, Denmark) and Merck (Darmstadt, Germany); consulting and advising activities with Eli Lilly and Company (Indianapolis, IN, USA); advising activities with Pfizer Inc. (New York, NY, USA), Sanofi (Paris, France), and Diabetes Technology Management (Foster City, CA, USA); royalties from UpToDate (Waltham, MA, USA); and speaker's forum with Medikinetics (San Diego, CA, USA).

\section{Published: 13 Nov 2013}

\section{References}

1. Christiansen CF, Johansen MB, Christensen S, O'Brien JM, T $\varphi$ nnesen E: Preadmission metformin use and mortality among intensive care patients with diabetes: a cohort study. Crit Care 2013, 17:R192.

2. Duncan Al, Koch CG, Xu M, Manlapaz M, Batdorf B, Pitas G, Starr N: Recent metformin ingestion does not increase in-hospital morbidity or mortality after cardiac surgery. Anesth Analg 2007, 104:42-50.

3. Umpierrez GE, Hellman R, Korytkowski MT, Kosiborod M, Maynard GA, Montori VM, Seley JJ, Van den Berghe G: Management of hyperglycemia in hospitalized patients in non-critical care setting: an endocrine society clinical practice guideline. J Clin Endocrinol Metab 2012, 97:16-38.

4. Moghissi ES, Korytkowski MT, DiNardo M, Einhorn D, Hellman R, Hirsch IB, Inzucchi SE, Ismail-Beigi F, Kirkman MS, Umpierrez GE, American Association of Clinical Endocrinologists, American Diabetes Association: American Association of Clinical Endocrinologists and American Diabetes Association consensus statement on inpatient glycemic control. Diabetes Care 2009, 32:1119-1131.

5. Glucophage/Glucophage XR [package insert]. Princeton, NJ: Bristol-Myers Squibb Company; January 2009 [http://packageinserts.bms.com/pi/pi_ glucophage.pdf]

6. Salpeter SR, Greyber E, Pasternak GA, Salpeter EE: Risk of fatal and nonfatal lactic acidosis with metformin use in type 2 diabetes mellitus. Cochrane Database Syst Rev 2010, 4, CD002967.

7. Scheen AJ, Paquot N: Metformin revisited: a critical review of the benefit-risk balance in at-risk patients with type 2 diabetes. Diabetes Metab 2013, 39:179-190.

8. Lipska KJ, Bailey CJ, Inzucchi SE: Use of metformin in the setting of mild-to-moderate renal insufficiency. Diabetes Care 2011, 34:1431-1437.

9. National Health System National Diabetes Inpatient Audit; [http://www. diabetes.nhs.uk/information_and_data/diabetes_audits/ national_diabetes_inpatient_audit/]

10. United States Pharmacopeial Convention: MEDMARX 5th anniversary data report. A chartbook of 2003 findings and trends 1999-2003. http://www.usp. org/products/medMarx/index.html?USP_Print.

11. Lipska KJ, Wang Y, Kosiborod M, Masoudi FA, Havranek EP, Krumholz HM, Inzucchi SE: Discontinuation of antihyperglycemic therapy and clinical outcomes after acute myocardial infarction in older patients with diabetes. Circ Cardiovasc Qual Outcomes 2010, 3:236-242. 
12. Carey M, Boucai L, Zonszein J: Impact of hypoglycemia in hospitalized patients. Curr Diab Rep 2013, 13:107-113.

13. Dungan KM, Braithwaite SS, Preiser J: Stress hyperglycemia. Lancet 2009 373:1798.

14. Schwartz S, DeFronzo RA: Is incretin-based therapy ready for the care of hospitalized patients with type 2 diabetes? The time has come for GLP-1 receptor agonists! Diabetes Care 2013, 36:2107-2011.

15. Umpierrez GE, Korytkowski M: Is incretin-based therapy ready for the care of hospitalized patients with type 2 diabetes? Insulin therapy has proven itself and is considered the mainstay of treatment. Diabetes Care 2013, 36:2112-2117.

$10.1186 / \mathrm{cc} 13107$

Cite this article as: Dungan: Hyperglycemia in the intensive care unit: is insulin the only option? Critical Care 2013, 17:1012 